\title{
Verifications of the Scattering Theory on Manifolds
}

\author{
M. Y. Youssif'1, Elhadi E. E. Dalam² \\ ${ }^{1}$ Department of Mathematics, College of Science, Taif University, Taif, Saudi Arabia \\ ${ }^{2}$ Department of Mathematics, College of Arts and Science in Al Mandag, Al Baha University, Al Baha, Saudi Arabia \\ Email: mokhtar@tu.edu.sa, adalam@bu.edu.sa
}

How to cite this paper: Youssif, M.Y. and Dalam, E.E.E. (2020) Verifications of the Scattering Theory on Manifolds. Advances in Pure Mathematics, 10, 645-657.

https://doi.org/10.4236/apm.2020.1011040

Received: October 25, 2020

Accepted: November 24, 2020

Published: November 27, 2020

Copyright $\odot 2020$ by author(s) and Scientific Research Publishing Inc. This work is licensed under the Creative Commons Attribution International License (CC BY 4.0).

http://creativecommons.org/licenses/by/4.0/

\begin{abstract}
Scattering theory plays the main role in the study of manifolds and the Laplacian spectrum. In this article, we process justifying the continuous Laplacian spectrum $\Delta_{g_{i}}$ and $\Delta_{h_{i}}$ on a complete Riemannian manifold. $\left(M, g_{i}\right)$ is categorized by the use of bounded curvature of the metric. In particular, the covariant derivative is limitedly considered as an application in the geodesic distance from a fixed point.
\end{abstract}

\section{Keywords}

Manifolds, Scattering theory, Spectrum

\section{Introduction}

A great number of researchers referred to the connection between, time-dependent, time-independent, Laplacian, manifold, wave operators, matrices, Riemannian metric, and Schrödinger equation linked to the theory of scattering.

For example, Itoa, K. and Skibsted, E. in [1] included time-dependent scattering theory along with allowed range perturbation and scattering by obstacles. The "independent" and "dependent" scattering by particles has been studied in appropriate single-particle, and examples of independent scattering are described by Michael I. Mishchenko, see [2]. The scattering theory for the Laplacian on symmetric spaces of a non-compact type in the frame work of Agmon-Hörmander has been updated by Koichi Kaizuka in [3]. Thierry Cazenave and Ivan Naumk in [4] modified scattering for the critical nonlinear Schrödinger equation. The exhibited conditions under which the stationary wave operators and the strong wave operators exist and coincide have been discussed by R. Tiedra de Aldecoa [5]. The scattering matrices for dissipative quantum system and 
Neumann maps have been studied by many authors see [6] [7]. Subsequently, Rainer Hempel, Olaf Post, and Ricardo Weder [8] obtained the existence and completeness of the wave operators for perturbations of the Riemannian metric for the Laplacian on a complete manifold of dimension.

In this paper, we follow the exact reviews and approaches of Werner Muller and Corm Salomonsen in [9] with a slight change. The current study contributes to the expansion of the knowledge in this field by addressing the scattering theory for the Laplacian spectrum $\left(\Delta_{g_{i}}\right.$ and $\left.\Delta_{h_{i}}\right)$ on the manifold with bounded curvature comparison dynamics.

Definition 1. Let $\beta:[0, \infty) \rightarrow \mathbb{R}$ be a positive, continuous, non-increasing function. Then $\beta$ is called a function of moderate decay, if it satisfies the following condition:

(i) $\sup _{x \in[1, \infty)} x \beta(x)<\infty$;

(ii) $\exists C_{\beta}>0: \beta(x+y) \geq C_{\beta} \beta(x) \beta(y), x, y \geq 1$

Further $\beta$ is called of sub-exponential decay if for any $c>0, \mathrm{e}^{c x} \beta(x) \rightarrow \infty$. As $x \rightarrow \infty$.

Definition 2. Let $\beta$ be a function of moderate decay. Two metrics $g, h \in M$ are said to be $\beta$-equivalent up to order $k$ if There exist $q \in M$ and $C>0$ such that for all $x \in M$ we have ${ }^{k}|g-h|_{g}(x) \leq C \beta\left(1+d_{g}(x, q)\right)$ holds. In this case, we write $g \sim_{\beta}^{k} h$.

Definition 3. Let $s>0$. For $s>\varepsilon \geq 0$ let $K_{\varepsilon}(M, g ; s) \in \mathbb{N} \bigcup\{\infty\}$ be the smallest number such that there exists a sequence $\left\{x_{i}\right\}_{i=1}^{\infty}$ such that sup \# $\left\{i \in \mathbb{N} \mid x \in B_{3 S+\varepsilon}\left(x_{i}\right)\right\} \leq K_{\varepsilon}(M, g ; s)$ Further, let $\stackrel{x \in M}{K}(M, g ; s)=K_{0}(M, g ; s)$ put

$$
k(M, g ; s)=1 .
$$

Definition 4. Let $(M, g)$ be a complete. Then $\Delta: C_{c}^{\infty}(M) \rightarrow L^{2}(M)$ is essentially self-adjoint and function $f(\sqrt{\Delta})$ can be defined by the spectral theorem for unbounded self-adjoint operators by $f(\sqrt{\Delta})=\int_{0}^{\infty} f(\lambda) \mathrm{d} E_{(\lambda)}$, where $\mathrm{d} E_{(\lambda)}$ is the projection spectral measure associated with $\sqrt{\Delta}$. Let $f \in L^{1}(\mathbb{R})$ be even and let $\hat{f}(\lambda)=\int_{-\infty}^{\infty} f(x) \cos (\lambda x) \mathrm{d} x$. Then $f(\sqrt{\Delta})$ can also be defined by

$$
f(\sqrt{\Delta})=\frac{1}{2 \pi} \int_{-\infty}^{\infty} \tilde{f}(\lambda) \cos (\lambda \sqrt{\Delta}) \mathrm{d} \lambda .
$$

Eichhorn, Proposition 2.1 in [10] has shown that $M$ can be endowed with a canonical topology given by a metrizable uniform structure. For a given Riemannian metric $g_{i}$ on $M$, denote by $\nabla^{g_{i}}$ the Levi-Civita connection 2.5 in [11] of $g$ and by $\|0\|_{g}$ the norm induced by $g$ in the fibers of $\oplus_{p, q \geq 0}\left(T M \otimes T^{\star} M^{\otimes q}\right)$. Let $h$ be any other Riemannian metric on $M$. For $k \geq 0$ set

$$
\begin{aligned}
& \sum_{i=1}^{\infty}{ }^{k}\left|g_{i}-h_{i}\right|_{g_{i}}(x) \\
& =\sum_{i=1}^{\infty}\left(\left|g_{i}-h_{i}\right|_{g}(x)\right)+\sum_{j=0}^{k-1}\left(\left|\left(\nabla^{g}\right)^{j}\left(\nabla^{g}-\nabla^{h}\right)\right|(x)\right), x \in M,
\end{aligned}
$$


and $\sum_{i=1}^{\infty}\left({ }^{k}\left\|g_{i}-h_{i}\right\|_{g_{i}}\right)=\sup _{x \in M}{ }^{k} \sum_{i=1}^{\infty}\left(\left|g_{i}-h_{i}\right|_{g_{i}}(x)\right)$. Recall that two metrics $g_{i}, h_{i}$ are said to be quasi-isometric if there exist $C_{1}, C_{2}>0$ such that

$$
C_{1 g}(x) \leq \sum_{i=1}^{\infty} h_{i}(x) \leq C_{2 g}(x), \text { for all } x \in M
$$

in the sense of positive definite quadratic forms. We shall write $g_{i} \sim h_{i}$ for quasi-isometric metrics $g_{i}$ and $h_{i}$. If $g$ and $h$ are quasi-isometric, then (4) implies that for all $p, q \geq 0$, there exist $A_{P, q} B_{p, q}>0$ such that for every tensor field $T$ on $M$ of bidegree $(p, q)$ we have

$$
A_{p, q}|T|(x) \leq \sum_{i=1}^{\infty}|T|_{h_{i}}(x) \leq B_{p, q}|T|_{g}(x), x \in M
$$

\section{Theorems and Lemmas}

Lemma 1. Let $\beta$ be of moderate decay. Then there exist a constants $C>0$ and $c>0$ such that,

$$
\beta(x) \geq C \mathrm{e}^{-c x}, x \in[1, \infty)
$$

Lemma 2. Let $g, h \in C M$ be quasi-isometric. For every $k \geq 0$, there exists a polynomial $P_{k}\left(X_{1}, \cdots, X_{k}\right)$ depending on the quasi-isometry constants, with nonnegative coefficients and vanishing constant term, such that

$$
\begin{aligned}
& { }^{k}|g-h|_{h}(x) \\
& \leq P_{k}\left(|g-h|_{g}(x),\left|\nabla^{g}-\nabla^{h}\right|_{g}(x), \cdots,\left|\left(\nabla^{g}\right)^{k-1}\left(\nabla^{g}-\nabla^{h}\right)\right|_{g}(x)\right), x \in M
\end{aligned}
$$

Proof. From (4) follows that $|g-h|_{h}(x) \leq C_{3}|g-h|_{h}(x)$ and

$$
\left|\nabla^{g}-\nabla^{h}\right|_{h}(x) \leq C_{4}\left|\nabla^{g}-\nabla^{h}\right|_{g}(x), x \in M .
$$

This is as important as the first two terms in (3) and deals with the question for $k=0,1$. Now we shall proceed by induction. Let $k \geq 2$ and suppose that the lemma holds for $l \leq k-1$. For each, $p \leq 0$ we have

$$
\left(\nabla^{h}\right)^{p}\left(\nabla^{h}-\nabla^{g}\right)=\nabla^{g}\left(\nabla^{h}\right)^{p-1}\left(\nabla^{h}-\nabla^{g}\right)+\left(\nabla^{h}-\nabla^{g}\right)\left(\nabla^{h}\right)^{p-1}\left(\nabla^{h}-\nabla^{g}\right)
$$

Let $p \leq k$ using (7), (6) and the hypothesis, we can estimate the point wise $h$ norm the second term on the right-hand side of (8) in desired way deal with the first term. We use the formula

$$
\begin{aligned}
& \left(\nabla^{g}\right)^{p}\left(\nabla^{h}\right)^{l}\left(\nabla^{h}-\nabla^{g}\right) \\
& =\left(\nabla^{g}\right)^{(p+1)}\left(\nabla^{h}\right)^{(l-1)}\left(\nabla^{h}-\nabla^{g}\right)+\left(\nabla^{g}\right)^{p}\left(\nabla^{h}-\nabla^{g}\right)\left(\nabla^{h}\right)^{(l-1)}\left(\nabla^{h}-\nabla^{g}\right) .
\end{aligned}
$$

Applying the Leibniz rule, we get

$$
\begin{aligned}
& \left|\left(\nabla^{g}\right)^{p}\left(\nabla^{h}-\nabla^{g}\right)\left(\nabla^{h}\right)^{(l-1)}\left(\nabla^{h}-\nabla^{g}\right)\right|_{g}(x) \\
& \leq C \sum_{i=0}^{p}\left|\left(\left(\nabla^{g}\right)^{i}\left(\nabla^{h}-\nabla^{g}\right)\right)\right|_{g}(x) \cdot\left|\left(\left(\nabla^{g}\right)^{(p-i)}\left(\nabla^{h}\right)^{(l-1)}\left(\nabla^{h}-\nabla^{g}\right)\right)\right|_{g}(x)
\end{aligned}
$$


for some $C>0$ and all $x \in M$.Inserting (8) and iterating these formulas reduces everything to the induction hypothesis.

Lemma 3. Let $\beta$ be a function of moderate decay. Then for all $x, y, q \in M$, we have

$$
C_{\beta} \beta(1+d(x, y)) \leq \frac{\beta(1+d(x, q))}{\beta(1+d(y, q))} \leq \frac{1}{C_{\beta} \beta(1+d(x, y))}
$$

Moreover, for every $q^{\prime} \in M$ there exists a constant $C>0$, depending only on $q$ and $q^{\prime}$ such that

$$
C^{-1} \beta\left(1+d\left(x, q^{\prime}\right)\right) \leq \beta(1+d(x, q)) \leq \beta\left(1+d\left(x, q^{\prime}\right)\right) .
$$

Lemma 4. There exists a constant $C>0$ depend only on $K$ such that

$$
\tilde{i}(x) \geq C \tilde{i}(p)^{n} \mathrm{e}^{-(n-1) \sqrt{K} d(x, p)}
$$

for all $x, p \in M$.

Lemma 5. For $r \leq \tilde{i}\left(x_{0}\right)$,

$\frac{2 \pi^{\frac{n}{2}}}{\Gamma\left(\frac{n}{2}\right)} \int_{0}^{r}\left(\frac{\sin t \sqrt{K}}{\sqrt{K}}\right)^{(n-1)} \mathrm{d} t \leq \operatorname{VOL}\left(B_{r}\left(x_{0}\right)\right) \leq \frac{2 \pi^{\frac{n}{2}}}{\Gamma\left(\frac{n}{2}\right)} \int_{0}^{r}\left(\frac{\sin h \sqrt{K}}{\sqrt{K}}\right)^{(n-1)} \mathrm{d} t$

We note that the inequality on the right-hand side holds for all $r \in \mathcal{R}$. In particular $\operatorname{Vol}\left(B_{r}\left(x_{0}\right)\right)=0\left(\mathrm{e}^{(n-1) \sqrt{K r}}\right)$ as $r \rightarrow \infty$.

It is also important to know the maximal possible decay of the injectivity radius.

Lemma 6. $k_{\varepsilon}(M, g ; s)$ finite for all $s>\varepsilon$. Moreover, there exist constants $C, C>0$, which depend only on $K$, such that for $s>\frac{2 \pi}{\sqrt{K}}+\varepsilon$, we have $k_{\varepsilon}(M, g ; s) \leq C \mathrm{e}^{c s}$.

Lemma 7. Let $k \geq 1$ be even. Assume that $M$ has bounded curvature of order $k$. Let $k>0$ be such that $\sup _{x \in M} \sum_{l=0}^{2 k}\left|\nabla^{l} R(x)\right| \leq k$, there exist constants $r_{0}=r_{0}(k)>0$ and $C=C(k)>0$ such that for all $x_{0} \in M$ and $r_{i} \leq \min \left\{r_{0}, \tilde{r}\left(x_{0}\right)\right\} \quad$ one has $\sum_{i=1}^{\infty}\left\|u_{i}\right\|_{W^{2 K}\left(B_{r_{i}}\left(x_{0}\right)\right)} \leq C \sum_{i=1}^{\infty}\left\|u_{i}\right\|_{H^{2 k}\left(B_{r_{i}}\left(x_{0}\right)\right)}$ for all $u_{i} \in C_{0}^{\infty}\left(B_{r_{i}}\left(x_{0}\right)\right)$.

Lemma 8. Let $k \in \mathbb{N}$ be even. Suppose that $\left(M, g_{i}\right)$ has bounded curvature of order $2 k$ Let $\beta: M \rightarrow \mathbb{R}^{+}$be a function of moderate decay. Then there exists a canonical bounded inclusions $H_{\beta_{\tau}{ }^{\alpha-2 k n}}^{k}(M) \rightarrow W_{\beta}^{k}(M)$ and $H_{\beta}^{k}(M) \rightarrow W_{\beta \tau^{2 k k n}}^{k}(M)$

Proof. By Theorem (2.6) in [9] in M there exist a covering $\sum_{i=1}^{\infty} B_{\frac{\tilde{\tau}}{2^{k}}\left(x_{i}\right)}\left(x_{i}\right)$ of $M$ by balls and a constant $C>0$ such that

$$
\forall x \in M:\left\{x_{i} \mid x \in \sum_{i=1}^{\infty} B_{\tilde{\tau}\left(x^{i}\right)}\left(x_{i}\right)\right\} \leq C
$$

Let $\varphi \in C^{\infty}(\mathbb{R})$ go be such that $\varphi=1$ on $[0,1]$ and $\varphi=0$ on $[2, \infty)$ for 
$x \in M$ and $1 \leq j \leq k$, we define

$$
\sum_{j=1}^{k} \varphi_{j, x}(y)= \begin{cases}\sum_{j=1}^{k} \varphi\left(2^{j} \frac{d(x, y)}{\tilde{\tau}(x)}\right), & y \in B_{\tilde{\tau}(x)}(x) \\ 0, & \text { otherwise. }\end{cases}
$$

then $\sum_{j=1}^{k} \varphi_{j, x} \in C_{0}^{\infty}(M)$. Let $f \in H^{k}(M)$. Using Lemma 6, it follows that $\varphi_{j, x} f \in H^{k}\left(B_{\tilde{\tau}_{x}}(x)\right)$. Then by Lemma 7 , we get $\varphi_{j, x} f \in W^{k}\left(B_{\tilde{\tau}_{x}}(x)\right)$ and by the Leibniz rule there is $C>0$ such that

$$
\left|\sum_{j=1}^{k} \nabla^{j}\left(\varphi_{k, x} f\right)\right|_{g}(y) \leq C \sum_{p=0}^{j}\left|\nabla^{p} \varphi_{k, x}\right|_{g}(y) \cdot\left|\nabla^{j-p} f\right|_{g}(y), y \in M
$$

By estimating the supremum-norm of the derivatives of $\varphi_{k, x}$ and using Lemma 7, we get

$$
\begin{aligned}
\left\|\varphi_{k, x} f\right\|_{W^{k}} & \leq C\|f\|_{W^{k}}\left(B_{\frac{\tau}{2^{k-1}(x)}}(x)\right)+C^{\prime} \sum_{p=1}^{k}\left(\begin{array}{c}
k \\
p
\end{array}\right)(x)\left\|\varphi_{k-1, x} f\right\|_{W^{k-p}} \\
& \leq C\|f\|_{H^{k}}\left(B_{\frac{\tilde{\tau}}{2^{k-1}(x)}}(x)\right)+C^{\prime \prime} \sum_{p=1}^{k}\left(\begin{array}{l}
k \\
p
\end{array}\right) \tilde{\tau}^{-p}(x)\left\|\varphi_{k-1, x} f\right\|_{H^{k-p}}
\end{aligned}
$$

By induction, this yields

$$
\left\|\varphi_{k, x_{i}} f\right\|_{W^{k}} \leq C \tilde{\tau}^{-K}\left(x_{i}\right)\|f\|_{H^{k}}\left(B_{\frac{\tilde{\tau}}{2^{k-1}}(x)}(x)\right)+C^{\prime} \sum_{p=1}^{k}\left(\begin{array}{c}
k \\
p
\end{array}\right) \tilde{\tau}^{-P}(x)\left\|\varphi_{k-1, x} f\right\|_{W^{k-p}}
$$

Let $f \in H_{\beta}^{k}$. By Lemma 7, (11) and (12) we get

$$
\begin{aligned}
\|f\|_{W_{\beta}^{k}} & \leq C \sum_{i=1}^{\infty} \beta^{\frac{1}{2}}\left(x_{i}\right)\left\|\varphi_{k, x_{i}} f\right\|_{W^{k}} \tilde{\tau}^{k}\left(x_{i}\right) \leq C \sum_{i=1}^{\infty} \beta^{\frac{1}{2}}\left(x_{i}\right)\left\|\varphi_{k, x_{i}} f\right\|_{H^{k}} \\
& \leq C \sum_{i=1}^{\infty} \beta^{\frac{1}{2}}\left(x_{i}\right) \tilde{\tau}^{-k}\left(x_{i}\right)\|f\|_{H^{k}\left(\beta_{\tau\left(x_{i}\right)}(x)\right)}
\end{aligned}
$$

By (10) there exists $C_{1}>0$ such that $\tilde{\tau}\left(x_{i}\right)^{-k} \tilde{\tau}(x)^{k n} \leq C_{1}$ for all $i \in \mathbb{N}$ and $x \in B_{\tau\left(x_{i}\right)}\left(x_{i}\right)$. This implies $\sum_{i=1}^{\infty} \beta^{\frac{1}{2}}\left(x_{i}\right) \tilde{\tau}^{-k}\left(x_{i}\right)\|f\|_{H^{k}\left(B_{\tau\left(x_{i}\right)}\left(x_{i}\right)\right)} \leq C_{2}\|f\|_{H_{\tilde{\tau}^{-2 k n} \beta}^{k}}$. Assume that $(M, g)$ is complete. Then $\Delta: C_{0}^{\infty}(M) \rightarrow L^{2}(M)$ is essentially self-ad joint and function $f(\sqrt{\Delta})$ can be defined by the spectral theorem for unbounded self-ad joint operators by $f(\sqrt{\Delta})=\int_{0}^{\infty} f(\lambda) \mathrm{d} E_{\lambda}$, where $\mathrm{d} E_{\lambda}$ is the projection spectral measure associate with $\sqrt{\Delta}$. Let $f \in L^{1}(\mathbb{R})$ be even and let $\tilde{f}(\lambda)=\int_{-\infty}^{\infty} f(x) \cos (\lambda x) \mathrm{d} x$, then $f(\sqrt{\Delta})$ can also be defined by

$$
f(\sqrt{\Delta})=\frac{1}{2 \pi} \int_{-\infty}^{\infty} \tilde{f}(\lambda) \cos (\lambda \sqrt{\Delta}) \mathrm{d} \lambda
$$

This representation has been used in [12] to study the kernel of $f(\sqrt{\Delta})$ we will used (13) to study $f(\sqrt{\Delta})$ as operator in weighted $L^{2}$-spaces. To this end we need to study $\cos (\lambda \sqrt{\Delta})$ as operator in $L_{\beta}^{2}(M)$ given $s>0$, let $K(M, g ; s)$ be the constant introduced in Definition (1.3). 
Theorem 1. Assume that $(M, g)$ has bounded curvature. Let $\beta$ be a function of moderate decay. Then $\cos (\lambda \sqrt{\Delta})$ extends to a bounded operator in $L_{\beta}^{2}(M)$ for all $s \in \mathbb{R}$ and there exist $C, c>0$, such that

$$
\|\cos (s \sqrt{\Delta})\|_{L_{\beta}^{2}, L_{\beta}^{2}} \leq C \mathrm{e}^{c|s|}, s \in \mathbb{R} .
$$

Moreover $\cos (s \sqrt{\Delta}): L_{\beta}^{2}(M) \rightarrow L_{\beta}^{2}(M)$ is strongly continuous in $S$.

Proof. Let $s>0$ Choose a sequence $\left\{X_{k}\right\}_{k=1}^{\infty} \subset M$ which minimizes. $\kappa\left(M, g_{i} ; s\right)$. For $k \in \mathbb{N}$ let $P_{k}$ denote the multiplication by the characteristic function of $B_{s}\left(x_{k}\right) \backslash \bigcup_{i=0}^{k-1} B_{s}\left(x_{i}\right)$. Then each $P_{k}$ is an orthogonal projection in $L^{2}(M)$ and $L_{\beta}^{2}(M)$ respectively. Moreover the projections satisfy $P_{k} P_{k^{\prime}}=0$ for $k \neq k^{\prime}$ and $\sum_{k=1}^{\infty} P_{k}=1$ where the series is strongly convergent. Obviously the image of $P_{k}$ consists of functions with support in $B_{s}\left(x_{k}\right)$. Now recall that $\cos (\tau \sqrt{\Delta})$ has unit propagation speed [13], i.e., $\sup p \cos (\tau \sqrt{\Delta}) \delta_{s} \subset \overline{B_{|\tau|}(x)}$ for all $x \in M$ and $\tau \in \mathbb{R}$. Let $f \in L^{2}(M)$. Then it follows that $\sup p \cos (s \sqrt{\Delta}) P_{k} f \subset B_{2 s}\left(x_{k}\right)$ and $\sup p \cos (s \sqrt{\Delta})\left(\left(1-\chi_{B_{3 s}\left(x_{k}\right)}\right) f\right) \subset M-B_{2 s}\left(x_{k}\right)$ Hence

$$
\begin{aligned}
\|\cos (s \sqrt{\Delta}) f\|_{\beta}^{2} & =\sum_{k=1}^{\infty}\left\langle\cos (s \sqrt{\Delta}) P_{k} f, \cos (s \sqrt{\Delta}) f\right\rangle_{\beta} \\
& =\sum_{k=1}^{\infty}\left\langle\cos (s \sqrt{\Delta}) P_{k} f, \cos (s \sqrt{\Delta})\right\rangle
\end{aligned}
$$

Now observe that the norm of $(s \sqrt{\Delta})$ as an operation in $L^{2}(M)$ is bounded by 1 . This implies

$$
\left|\left\langle\cos (s \sqrt{\Delta}) P_{k} f, \cos (s \sqrt{\Delta})\left(\chi_{B_{3 s}\left(x_{k}\right)} f\right)\right\rangle\right| \leq \sup _{y \in B_{3 s}\left(x_{k}\right)} \beta(y)\left\|P_{k} f\right\|_{L^{2}} \cdot\left\|\chi_{B_{3 s}\left(x_{k}\right)} f\right\|_{L^{2}}
$$

To estimate the right-hand side, we write

$$
\sup _{y \in B_{3 s}\left(x_{k}\right)} \beta(y)\left\|P_{k} f\right\|_{L^{2}}^{2} \leq C_{\beta}^{-1} \frac{1}{\beta(1+4 s)}\left\|P_{k} f\right\|_{L_{\beta}^{2}}^{2}
$$

Since the support of $P_{k} f$ is contained in $B_{s}\left(x_{k}\right)$ we can use (9) to estimate the right-hand side. This gives $\sup _{y \in B_{3 s}\left(x_{k}\right)} \beta(y)\left\|P_{k} f\right\|_{L^{2}}^{2} \leq C_{\beta}^{-1} \frac{1}{\beta(1+4 s)}\left\|P_{k} f\right\|_{L_{\beta}^{2}}^{2}$. A similar inequality holds with respect to $\left\|\chi_{B_{3 s}}\left(x_{k}\right) f\right\|_{L^{2}}$ putting the estimations together, we get

$$
\left|\left\langle\cos (s \sqrt{\Delta}) P_{k} f, \cos (s \sqrt{\Delta})\left(\chi_{B_{3 s}\left(x_{k}\right)} f\right)\right\rangle\right| \leq C_{\beta}^{-1} \frac{1}{\beta(1+6 S)}\left\|P_{k} f\right\|_{L_{\beta}^{2}}\left\|\chi_{B_{3 s}\left(x_{k}\right)} f\right\|_{L_{\beta}^{2}}
$$

Now recall that by Lemma 6 , we have $\kappa(M, g ; s)<\infty$. Hence together with (14) and (15) we obtain

$$
\|\cos (s \sqrt{\Delta}) f\|_{L_{\beta}^{2}}^{2} \leq C_{\beta}^{-1} \frac{1}{\beta(1+6 s)}\|f\|_{\beta}^{2} \sum_{k=1}^{\infty}\left\|\chi_{B_{3 s}\left(x_{k}\right)} f\right\|_{L_{\beta}^{2}} \leq C_{\beta}^{-1} \frac{1}{\beta(1+6 s)} \kappa(M, g, s)^{\frac{1}{2}}\|f\|_{L_{\beta}^{2}}^{2}
$$


Recall that by (1) we have $\beta(x) \leq C(1+d(x, p))^{-1}, x \in M$. Therefore, $L^{2}(M) \subset L_{\beta}^{2}(M)$, and $L^{2}(M)$ is a dense subspace of $L_{\beta}^{2}(M)$. This implies that $\cos (s \sqrt{\Delta})$ extends to a bounded operator in $L_{\beta}^{2}(M)$. Moreover by (7) and Lemma 6, it follows that there exist constants $C, C>0$ such that $\|\cos (s \sqrt{\Delta})\|_{L_{\beta}^{2}, L_{\beta}^{2}}^{2} \leq C \mathrm{e}^{c s}, s \in[0, \infty)$. Since $\cos (-s \sqrt{\Delta})=\cos (s \sqrt{\Delta})$ this extends to all $s \in \mathbb{R}$ such that holds. The strong continuity is a consequence of the local bound of the norm and the strong continuity on the dense subspace $\cos (-s \sqrt{\Delta}) L^{2}(M) \subseteq L_{\beta}^{2}(M)$. Using Theorem 1 , we can study $f(\sqrt{\Delta})$ as an operator in $L_{\beta}^{2}(M)$ given $c \geq 0$, let $\mathcal{F}^{\prime}(c)=\left\{f \in L^{1}(\mathbb{R}): \int_{-\infty}^{\infty}|\tilde{f}(\lambda)| \mathrm{e}^{c|\lambda| \mathrm{d} \lambda<\infty}\right\}$.

Lemma 9. Let $\beta$ a function of moderate decay. If $\lambda$ and $\bar{\lambda}$ satisfy conditions (b) of Corollary 4.3 in [9] then

$$
H_{\beta}^{2}(M)=(\Delta-\lambda)^{-1}\left(L_{\beta}^{2}(M)\right) .
$$

Proof. First: note that $C_{0}^{\infty}(M)$ is dense in $L_{\beta}^{2}(M)$. Indeed $C_{0}^{\infty}(M)$ is dense in $L^{2}(M)$ and $L^{2}(M)$ is dense in $L_{\beta}^{2}(M)$. Let $f=\sum_{i=1}^{\infty}(\Delta-\lambda)^{-1} g_{i}, g_{i} \in L_{\beta}^{2}(M)$. Then there exists a sequence $\left\{\varphi_{i}\right\}_{i \in \mathbb{N}} \subset C_{0}^{\infty}(M)$ which converges to $\sum_{i=1}^{\infty} g_{i}$ in $L_{\beta}^{2}(M)$ and $(\Delta-\lambda)^{-1} \varphi_{i}$ converges to $f$ in $L^{2}(M)$. Let $\varphi \in L_{0}^{\infty}(M)$. Then

$$
\langle f, \Delta \varphi\rangle=\lim _{i \rightarrow \infty}\left\langle(\Delta-\lambda)^{-1}, \Delta \varphi\right\rangle=\lim _{i \rightarrow \infty}\left\langle\varphi_{i}+\lambda(\Delta-\lambda)^{-1} \varphi_{i}, \varphi\right\rangle=\langle g+\lambda f, \varphi\rangle .
$$

Thus $\Delta f=\sum_{i=1}^{\infty}\left(g_{i}+\lambda f\right) \in L_{\beta}^{2}(M)$ and hence $f \in L_{\beta}^{2}(M)$ now suppose that $f \in L_{\beta}^{2}(M)$ and set $g=(\Delta-\lambda) f$. Then $g \in L_{\beta}^{2}(M)$ and we need to show that $f=\sum_{i=1}^{\infty}(\Delta-\lambda)^{-1} g_{i}$. Let $\varphi \in C_{0}^{\infty}(M)$. By definition of $\sum_{i=1}^{\infty}(\Delta-\lambda)^{-1} g_{i}$, there exists a sequence $\{g\}_{i \in \mathbb{N}} \subset L^{2}(M)$ such that $(\Delta-\lambda)^{-1} g_{i}$ converges to $(\Delta-\lambda)^{-1} g$ in $L_{\beta}^{2}(M)$ as $i \rightarrow \infty$. Using this fact, we get

$\left\langle\sum_{i=1}^{\infty}(\Delta-\lambda)^{-1} g_{i}, \sum_{i=1}^{\infty} \varphi_{i}\right\rangle=\left\langle g_{i},(\Delta-\bar{\lambda})^{-1} \sum_{i=1}^{\infty} \varphi_{i}\right\rangle=\left\langle(\Delta-\lambda) f,(\Delta-\bar{\lambda})^{-1} \varphi\right\rangle$.

Now, observe that $(\Delta-\lambda)^{-1} \sum_{i=1}^{\infty} \varphi_{i}$ belongs to $H^{2}(M)$. By Lemma (3.1) in [9] there exists a sequence $\sum_{i=1}^{\infty} \varphi_{i} \subset(M)$ which converges to $(\Delta-\bar{\lambda})^{-1}$ in $H^{2}(M)$. Thus

$$
\begin{aligned}
& \left\langle(\Delta-\lambda) f,(\Delta-\bar{\lambda})^{-1} \varphi\right\rangle=\lim _{i \rightarrow \infty}\left\langle(\Delta-\lambda) f, \sum_{i=1}^{\infty} \varphi_{i}\right\rangle \\
& =\left\langle f,(\Delta-\bar{\lambda}) \sum_{i=1}^{\infty} \varphi_{i}\right\rangle=\langle f, \varphi\rangle .
\end{aligned}
$$

Together with (16) this implies that $(\Delta-\lambda)^{-1} g_{i}$.

Lemma 10. Let $\beta$ be of moderate decay. Assume that $g_{i} \sim_{\beta}^{k} h_{i}$ then the Sobolev spaces $W_{\xi}^{k}\left(M ; g_{i}\right)$ and $W_{\xi}^{k}\left(M ; h_{i}\right)$ are equivalent.

Proof. First note that by Lemma 1.7 in [9] the metrics $g$ and $h$ are quasi-isometric. This implies that $L_{\xi}^{2}\left(M ; g_{i}\right)$ and $L_{\xi}^{2}\left(M ; h_{i}\right)$ are equivalent. So the statement of the lemma holds for $k=0$. Let $f \in C^{\infty}(M)$ and $k \in \mathbb{N}$ by induction we will prove that for $l \leq k$ there exists $C_{1}>0$ such that for 


$$
\begin{aligned}
a, b \in \mathbb{N}_{0}, & a+b=l, \\
& \quad\left|\left(\nabla^{g_{i}}\right)^{a}\left(\nabla^{h_{i}}\right)^{b} f\right|_{h_{i}}(x) \leq C_{l} \sum_{i=0}^{(a+b)}\left|\left(\nabla^{g}\right)^{i} f\right|_{(x)}(x), x \in M .
\end{aligned}
$$

Let $l=1$. Since on functions the connections equal, (17) follows from quasi-isometry of $g$ and $h_{i}$. Next suppose that (17) holds for $1 \leq l<k$. To establish (17) for $l+1$, we proceed by induction with respect to $a$. Let $a, b \in \mathbb{N}_{0}$ with $a+b=l+1$. We may assume that $a<l+1$. Using

$$
\sum_{i=1}^{\infty}\left(\nabla^{g_{i}}\right)^{a}\left(\nabla^{h_{i}}\right)^{b} f=\sum_{i=1}^{\infty}\left(\nabla^{g_{i}}\right)^{a}\left(\nabla^{h_{i}}-\nabla^{g_{i}}\right)\left(\nabla^{h_{i}}\right)^{(b-1)} f+\left(\nabla^{g_{i}}\right)^{(a+1)}\left(\nabla^{h_{i}}\right)^{(b-1)} f,
$$

and $g \sim_{\beta}^{k} h$, it follows that (17) holds for $l+1$. Especially, putting $a=0$ we get

$$
\sum_{i=1}^{\infty}\left|\left(\nabla^{h_{i}}\right)^{l} f\right|_{h_{i}}(x) \leq C_{l} \sum_{i=0}^{l}\left|\left(\nabla^{g}\right)^{i} f\right|_{g_{i}}(x), x \in M, l \leq k .
$$

Suppose that $f \in C^{\infty}(M) \cap W_{\xi}^{k}\left(M ; g_{i}\right)$ then (18) implies that $f \in C^{\infty}(M) \cap W_{\xi}^{k}\left(M ; h_{i}\right)$ and $\|f\|_{W_{\xi}^{k}\left(M ; h_{i}\right)} \leq C\|f\|_{W_{\xi}^{k}\left(M ; g_{i}\right)}$.

By Lemma (3.1) in [9] $C^{\infty}(M) \cap W_{\xi}^{k}\left(M ; g_{i}\right)$ is dense in $W_{\xi}^{k}\left(M ; g_{i}\right)$. Therefore this inequality holds for all $f \in C^{\infty}\left(M, g_{i}\right)$. By symmetry, a similar inequality holds with the roles of $g_{i}$ and $h_{i}$ inter-changed. This concludes the proof.

Next we compare the Sobolev spaces $H_{\xi}^{2 k}\left(M ; g_{i}\right)$ and $H_{\xi}^{2 k}\left(M ; h_{i}\right)$. Let $\Delta_{g_{i}}$ denote the Laplace operator with respect to the metric $g$. Recall, that $\sum_{i=1}^{\infty} \Delta_{g_{i}}=\sum_{i=1}^{\infty}\left(\nabla^{g_{i}}\right)^{*} \nabla^{g_{i}}$, and that the formal ad joint $\left(\nabla^{g_{i}}\right)^{*}$ of $\left(\nabla^{g_{i}}\right)$ is given by $\left(\nabla^{g}\right)^{*}=-\operatorname{Tr}\left(g^{-1} \nabla^{g_{i}}\right)$. Where $\sum_{i=1}^{\infty} \Delta_{g_{i}}=\sum_{i=1}^{\infty}\left(\nabla^{g_{i}}\right)^{*} \nabla^{g_{i}}$ is the isomorphism induced by the metric and $\operatorname{Tr}: T^{*} M \otimes T M \rightarrow \mathbb{R}$ denotes $\sum_{i=1}^{\infty} \Delta_{g_{i}}=\sum_{i=1}^{\infty}\left(\nabla^{g_{i}}\right)^{*} \nabla^{g_{i}}$ contraction. Since contraction commutes with covariant differentiation and $\nabla^{g_{i}} g_{i}^{-1}=0$, we get the well-known formula $\Delta=-\operatorname{Tr}\left(g^{-1} \nabla^{2}\right)$. This can be iterated. For $\omega_{1} \otimes \cdots \otimes \omega_{k} \in\left(T^{*} M\right)^{\otimes k}$ define $g_{j}^{-1}\left(\omega_{1} \otimes \cdots \otimes \omega_{k}\right): \omega_{1} \otimes \cdots \otimes \omega_{j-1} \otimes g^{-1}\left(\omega_{j}\right) \otimes \omega_{j+1} \otimes \cdots \otimes \omega_{k}$, and let $\operatorname{Tr}_{i, j}\left(g_{j}^{-1}\right)$ denote, $\left(g_{j}^{-1}\right)$ followed by the contraction of the $i$ th and $t$ th component using. That contraction commutes with covariant differentiation and $\nabla^{g_{i}} g_{i}^{-1}=0$, we get

$$
\Delta_{g}^{k}=(-1)^{k} \operatorname{Tr}_{1,2}\left(g_{2}^{-1}\right) \circ \cdots \circ \operatorname{Tr}_{2 k-1,2 k}\left(g_{2 k}^{-1}\right)\left(\nabla^{g}\right)^{2 k} .
$$

In more traditional notation this mean $\Delta_{g_{i}}^{k} f=(-1)^{k} \sum_{i_{1}, \cdots, i_{k}} f_{; i_{1} i_{1} i_{2} i_{2} \cdots i_{k} i_{k}}$. For short notation we will write $\operatorname{Tr}\left(\left(g^{-1}\right)^{\otimes k}\right):=\operatorname{Tr}_{1,2}\left(g_{2}^{-1}\right) \circ \cdots \circ \operatorname{Tr}_{2 k-1,2 k}\left(g_{2 k}^{-1}\right)$.

Lemma 11. Assume that $g_{i} \sim_{\beta}^{2 k} \beta$. Then for each $l, 0 \leq l \leq 2 k$ and 
$j, 0 \leq j \leq 2 l$, there exist section $\xi_{j l}^{g}, \xi_{j l}^{h} \in C^{\infty}\left(\operatorname{Hom}\left(T^{*} M\right)^{\otimes j}, \mathbb{R}\right)$ such that $\sum_{l=0}^{2 k}\left(\Delta_{g}^{l}-\Delta_{h}^{l}\right)=\sum_{j=0}^{2 l} \xi_{j l}^{g} \circ\left(\nabla^{g}\right)^{j}=\sum_{j=0}^{2 l} \xi_{j l}^{h} \circ\left(\nabla^{h}\right)^{j}$ and there exists $C<0$ such that for $0 \leq p \leq l, \quad \sum_{j=0}^{2 l}\left(\left|\left(\nabla^{g}\right)^{p} \xi_{j l}^{g}\right|(x)\right) \leq C \beta(x)$, $\sum_{j=0}^{2 l}\left(\left|\left(\nabla^{h}\right)^{p} \xi_{j l}^{h}\right|_{h}(x)\right) \leq C \beta(x), \quad x \in M$.

Lemma 12. Assume that $\beta$ is a function of moderate decay and there exist real numbers $a, b$ such that

(i) $b \geq 1$, and $a+b=2$,

(ii) $\beta^{\frac{b}{3}} \in L^{1}(M)$,

(iii) $\beta^{\frac{a}{3}} \tilde{\tau}^{-n(n+2)} \in L^{\infty}(M)$.

Let $M_{\beta}$ be the operator of multiplication by $\beta$. Then the operator all $M_{\tau-2 n} M_{\beta} \Delta^{p} \mathrm{e}^{-t \Delta}$ is a trace-class operator for $\beta \in \mathbb{N}$ and $t$ in a compact interval, the trace-class norm is bounded.

\section{Main Results}

The main verification results are the following corollaries and lemma.

Corollary 1. Let $K, \lambda>0$ be given. There exists $r_{0}=r_{0}(K, \lambda)>0$ and $C=C(\lambda)>0$ such that for all $r_{i} \leq r_{0}, \quad p \in \varepsilon l l^{m}\left(r_{i}, K, \lambda\right)$ and $x_{0} \in B_{r_{i}}$. $\sum_{i=1}^{n}\left\|u_{i}\right\|_{W^{m}\left(B_{r_{i}}\right)} \leq C \sum_{i=1}^{n}\left(\left\|P_{u_{i}}\right\|_{L^{2}\left(B_{r}\right)}+\left\|u_{i}\right\|_{L^{2}\left(B_{r_{i}}\right)}\right)$ for all $\sum_{i=1}^{n} u_{i} \in C_{0}^{\infty}\left(\sum_{i=1}^{n} B_{r_{i}}\right)$.

Proof. Let $1 \geq r_{i}>0$ and let $P \in \varepsilon l l^{m}\left(r_{i}, K, \lambda\right)$. Put $P_{0}=\sum_{|\alpha|=m} a_{\alpha}(0) D^{\alpha}$.By lemma 17.1.2 in [14] there exists $C_{1}>0$ which depends only on $\lambda$ such that for all $\sum_{i=1}^{n} u_{i} \in C_{0}^{\infty}\left(B_{r_{i}}\right)$ :

$$
\sum_{i=1}^{n}\left\|u_{i}\right\|_{W^{m}\left(B_{r_{i}}\right)} \leq C \sum_{i=1}^{n}\left(\left\|P_{0} u_{i}\right\|_{L^{2}\left(B_{r_{i}}\right)}+\left\|u_{i}\right\|_{L^{2}\left(B_{r_{i}}\right)}\right) .
$$

Now $\sum_{i=1}^{n} p u_{i}=\sum_{i=1}^{n} P_{0} u_{i}+\sum_{i=1}^{n}\left(P-P_{0}\right) u_{i}$. Thus

$\sum_{i=1}^{n}\left\|u_{i}\right\|_{W^{m}\left(B_{r_{i}}\right)} \leq C \sum_{i=1}^{n}\left(\left\|P u_{i}\right\|_{L^{2}\left(B_{r_{i}}\right)}+\left\|\left(P-P_{0}\right) u_{i}\right\|_{L^{2}\left(B_{r_{i}}\right)}+\left\|u_{i}\right\|_{L^{2}\left(B_{r_{i}}\right)}\right)$. Next observe that

$$
\sum_{i=1}^{n}\left(P-P_{0}\right) u_{i}=\sum_{i=1}^{n} \sum_{|\alpha|=m}\left(a_{\alpha}(x)-a_{\alpha}(0)\right) D^{\alpha} u_{i}+\sum_{i=1}^{n} \sum_{|\alpha|<m} a_{\alpha}(x) D^{\alpha} u_{i}
$$

Hence by lemma 17.1.2 in [14]:

$$
\begin{aligned}
& \sum_{i=1}^{n}\left\|\left(P-P_{0}\right) u_{i}\right\|_{L^{2}\left(B_{r_{i}}\right)} \\
& \leq \sum_{i=1}^{n} r_{i} \sum_{|\alpha|=m}\left\|a_{\alpha}\right\|_{C^{1}\left(B_{r_{i}}\right)}\left\|u_{i}\right\|_{W^{m}\left(B_{r_{i}}\right)}+\sum_{i=1}^{n} \sum_{|\alpha|<m}\left\|a_{\alpha}\right\|_{C^{O}\left(B_{r_{i}}\right)}\left\|u_{i}\right\|_{W^{m-1}\left(B_{r_{i}}\right)} \\
& \leq K \sum_{i=1}^{n}\left(r_{i}\left\|u_{i}\right\|_{W^{m}\left(B_{r_{i}}\right)}+\left\|u_{i}\right\|_{W^{m-1}\left(B_{r_{i}}\right)}\right)
\end{aligned}
$$

By the Poincare inequality there exists $C_{2}>0$ which is independent of $\sum_{i=1}^{n} r_{i} \leq 1$ such that for all $\sum_{i=1}^{n} u_{i} \in C_{0}^{\infty} \sum_{i=1}^{n}\left(B_{r_{i}}\right)$ : 
$\sum_{i=1}^{n}\left\|u_{i}\right\|_{W^{m-1}\left(B_{r_{i}}\right)} \leq \sum_{i=1}^{n} r_{i} C_{2}\left\|u_{i}\right\|_{W^{m}\left(B_{r_{i}}\right)}$. Using this inequality, it's follows from (21) that $\sum_{i=1}^{n}\left\|\left(P-P_{0}\right) u_{i}\right\|_{L^{2}\left(B_{r_{i}}\right)} \leq \sum_{i=1}^{n} r_{i} C(K)\left\|u_{i}\right\|_{W^{m}\left(B_{r_{i}}\right)}$. Together with (20) we get

$$
\sum_{i=1}^{n}\left(1-r_{i} C C(K)\right)\left\|u_{i}\right\|_{W^{m}\left(B_{r_{i}}\right)} \leq C \sum_{i=1}^{n}\left(\left\|P u_{i}\right\|_{L^{2}\left(B_{r_{i}}\right)}+\left\|u_{i}\right\|_{L^{2}\left(B_{r_{i}}\right)}\right)
$$

Set $r_{0}=\min \left\{1, \frac{1}{2 C C(K)}\right\}$ then it follows that for all $\sum_{i=1}^{n} r_{i} \leq r_{0}$ and

$$
\sum_{i=1}^{n} u_{i} \in C_{0}^{\infty} \sum_{i=1}^{n}\left(B_{r_{i}}\right): \sum_{i=1}^{n}\left\|u_{i}\right\|_{W^{m}\left(B_{r_{i}}\right)} \leq 2 C \sum_{i=1}^{n}\left(\left\|P u_{i}\right\|_{L^{2}\left(B_{r_{i}}\right)}+\left\|u_{i}\right\|_{L^{2}\left(B_{r_{i}}\right)}\right) .
$$

Corollary 2. Assume $\left(M, g_{i}\right)$ has bounded curvature and let $\beta$ be functions of moderate decay. Then there exists a constant $C=C\left(M, g_{i}, \beta\right)$ such that for all functions $f_{i} \in \mathcal{F}^{\prime}(c)$, the operator $f_{i}(\sqrt{\Delta})$ extends to abounded operator in $L_{\beta}^{2}(M)$. Moreover, there exists a constant $C_{1}=C_{1}\left(M, g_{i}, \beta\right)>0$ such that $\sum_{i=1}^{n}\left\|f_{i}(\sqrt{\Delta})\right\|_{L_{\beta}^{2}, L_{\beta}^{2}} \leq C_{1} \sum_{i=1}^{n}\|\hat{f}\|_{L_{\mathrm{e}^{1}}{ }^{\mathrm{c} \|}}$ for all $f_{i}$ as above. If $\kappa\left(M, g_{i} ; s\right)$ is at most sub-exponentially increasing, then $c\left(M, g_{i} ; \beta\right)>0$ can be chosen arbitrarily.

Proof. By Theorem 1, there exist constants $C, C>0$, depending on $\left(M, g_{i}, \beta\right)$ such that $\|\cos (\sqrt{\Delta})\|_{L_{\beta}^{2}, L_{\beta}^{2}} \leq C \mathrm{e}^{c||}$, for all $s \in \mathbb{R}$. Let $\varphi \in L^{2}(M)$ using (15), it follows that $\sum_{j=1}^{n}\left\|f_{j}(\sqrt{\Delta}) \varphi\right\|_{L_{\beta}^{2}} \leq \frac{C}{\sqrt{2 \pi}} \sum_{i=1}^{n}\left\|\hat{f}_{i}\right\|_{L^{1^{1} c \mid l}}$. Since $L^{2}(M)=L_{\beta}^{2}(M)$, it follows from (2) that $f(\sqrt{\Delta})$ extends to a bounded operator in $L_{\beta}^{2}(M)$. The last statement is obvious.

Corollary 3. Let $\beta$ be a function of moderate decay. Assume that there exist real numbers $a, b$ such that:

(i) $a+b=2$,

(ii) $\beta^{b} \in L^{1}(M)$,

(iii) $\beta^{a} \bar{t}^{-\frac{1}{2} n(n+1)} \in L^{\infty}(M)$.

Let $M_{\beta}$ the operator of multiplication by $\beta$. Then for every $p \in \mathbb{N}_{0}$ the operator $M_{\beta}\left(\sum_{i=1}^{n} \Delta_{g_{i}}^{p}\right) \mathrm{e}^{-t \Delta_{g_{i}}}$ is Hilbert-Schmidt. For $\mathrm{e}^{-t\left(\sum_{i=1}^{n} g_{i}\right)}$ in a compact interval in $\mathbb{R}^{+}$the Hilbert-Schmidt norm is bounded.

Proof. We have $M_{\beta} \Delta^{p} \mathrm{e}^{-t \Delta}=\left(M_{\beta} \mathrm{e}^{-\frac{1}{2} \Delta}\right)\left(\Delta^{p} \mathrm{e}^{\frac{-1}{2} \Delta}\right)$. Note that the operator norm of $\Delta^{P} \mathrm{e}^{-\frac{1}{2} \Delta}$ is bounded on compact subsets of $\mathbb{R}^{+}$. Hence we assume that $p=0$. Lemma 11, (i) implies that $\mathrm{e}^{-t \Delta} I \in L_{\beta^{b}}^{2}(M)$. Let $\mathrm{e}^{-t \Delta}(x, y)$ be the kernel $\mathrm{e}^{-t \Delta}$ then $\left\langle I, \mathrm{e}^{-t \Delta}\right\rangle_{L^{2}}=\int_{M} \int_{M} \prod_{i=1}^{n} \beta^{b}(x) \mathrm{e}^{-t \Delta_{g_{i}}}(x, y) \mathrm{d} y \mathrm{~d} x$. The integral converges since $\mathrm{e}^{-t \Delta}(x, y) \geq 0$ we get 


$$
\begin{aligned}
& \int_{M} \int_{M} \prod_{i=1}^{n}\left|\beta(x) \mathrm{e}^{-t \Delta_{g_{i}}}\right|^{2}(x, y) \mathrm{d} y \mathrm{~d} x \\
& =\int_{M} \int_{M} \prod_{i=1}^{n} \beta^{2}(x)\left(\mathrm{e}^{-t \Delta_{g_{i}}}(x, y)\right)^{2} \mathrm{~d} y \mathrm{~d} x \\
& \leq \sup _{z, w \in M}\left|\prod_{i=1}^{n} \beta^{a}(z) \mathrm{e}^{-t \Delta_{g_{i}}}(z, w)\right| \int_{M} \int_{M} \prod_{i=1}^{n} \beta^{b}(x) \mathrm{e}^{-t \Delta_{g_{j}}}(x, y) \mathrm{d} y \mathrm{~d} x \\
& \leq C \sup _{z \in M}\left|\beta^{a}(z) \tilde{t}^{-\frac{n(n+1)}{2}}(z)\right| \int_{M} \beta^{b}(x)\left(\mathrm{e}^{-t \Delta}(1)\right)(x) \mathrm{d} x \\
& \leq C_{1}\left\|\mathrm{e}^{-t \Delta}(1)\right\|_{L^{2}} .
\end{aligned}
$$

This proves the corollary.

Lemma 13. Let $\beta$ be a function of moderate decay, satisfying the conditions of Lemma 11. Let $g_{i}, h_{i}$ be two complete metrics on $M$ such that $g_{i} \sim_{\beta}^{2} h_{i}$. Let $\Delta_{g_{i}}$ and $\Delta_{h_{i}}$ be the Laplacians of $g_{i}$ and $h_{i}$, respectively. Then $\sum_{i=1}^{\infty}\left(\Delta_{g_{i}}-\Delta_{h_{i}}\right) \mathrm{e}^{-t \Delta_{g_{i}}}$ and $\sum_{i=1}^{\infty} \mathrm{e}^{-t \Delta_{g_{i}}}\left(\Delta_{g_{i}}-\Delta_{h_{i}}\right)$ are trace class operators, and the trace norm is uniformly bounded for $\tau$ in a compact subset of $(0, \infty)$.

Proof. We decompose $\mathrm{e}^{-\tau \Delta_{g_{i}}}$ as $\mathrm{e}^{-\tau \Delta_{g_{i}}}=\sum_{i=1}^{\infty}\left(\mathrm{e}^{-t \Delta_{g_{i}}} M_{\beta^{\frac{-1}{3}}}\right) \cdot\left(M_{\beta^{\frac{1}{3}}} \mathrm{e}^{\frac{-t}{2} \Delta_{g_{i}}}\right)$. By Lemma 11, the second factor is a Hilbert-Schmidt operator and it suffices to show that $\left(\Delta_{g_{i}}-\Delta_{h_{i}}\right) \mathrm{e}^{-t \Delta_{g}} M_{\beta^{-\frac{1}{3}}}$ is Hilbert-Schmidt and that the Hilbert-Schmidt norm is bounded for $t$ in a compact interval, using Lemmas 8 , and Lemmas 10, it follows that the Hilbert-Schmidt norm can be estimated by

$$
\begin{aligned}
& \sum_{i=1}^{\infty}\left(\left\|\left(\Delta_{g_{i}}-\Delta_{h_{i}}\right) \mathrm{e}^{-t \Delta_{g}} M_{\beta^{-\frac{1}{3}}}\right\|_{2}^{2}\right) \\
& \leq C \sum_{i=0}^{2} \int_{M} \int_{M}\left|\left(\nabla^{g}\right)^{i} \mathrm{e}^{-t \Delta_{g_{i}}}(x, y) \beta^{\frac{-1}{3}}(y)\right|_{g_{i}}^{2} \beta^{2}(x) \mathrm{d} x \mathrm{~d} y \\
& =C \sum_{i=0}^{\infty} \int_{M}\left\|\mathrm{e}^{t \Delta_{g_{i}}}(., y) \beta^{\frac{-1}{3}}(y)\right\|_{W_{\beta^{2}}^{2}}^{2} \mathrm{~d} y \\
& \leq C_{1} \sum_{i=0}^{\infty} \int_{M}\left\|\mathrm{e}^{t \Delta_{g_{i}}}(., y) \beta^{\frac{1}{3}}(y)\right\|_{H_{\beta_{t}^{2}-4 n}^{2}}^{2} \mathrm{~d} y \\
& \leq C_{2} \sum_{q=0}^{1} \int_{M}\left\|\beta(.) \tilde{t}^{-2 n}(.) \Delta_{g}^{q} \mathrm{e}^{-t \Delta_{g}}(., y) \beta^{\frac{-1}{3}}(y)\right\|_{2}^{2} \mathrm{~d} y \\
& =C_{2} \sum_{q=0}^{1} \int_{M}\left\|M_{\beta} M_{\tilde{i}^{-2 n}} \Delta_{g_{i}}^{q} \mathrm{e}^{-t \Delta_{g}} M_{\beta^{-\frac{1}{3}}}\right\|_{2}^{2} \mathrm{~d} y .
\end{aligned}
$$

By Lemma 13, the right-hand side is finite and bounded for $t$ in a compact interval of $\mathbb{R}^{+}$prove that $\sum_{i=1}^{\infty} \mathrm{e}^{-t \Delta_{g_{i}}}\left(\Delta_{g_{i}}-\Delta_{h_{i}}\right)$ is a trace class operator, it suffices to establish it for its adjoint $\sum_{i=1}^{\infty}\left(\Delta_{g_{i}}-\left(\Delta_{h_{i}}\right)^{*} g_{i}\right) \mathrm{e}^{-t \Delta_{g_{i}}}$ with respect to $t$. By 
(19) and (18) we have

$\sum_{i=1}^{\infty} \Delta_{g_{i}}\left(\Delta_{h_{i}}\right)^{* g}=\sum_{i=1}^{\infty}\left(\left(\xi_{01}^{g_{i}}\right)^{*_{g} g}+(\nabla)^{* g} \circ\left(\xi_{11}^{g_{i}}\right)^{* g}+\left[\left(\nabla^{g_{i}}\right)^{*_{g}}\right]^{2} \circ\left(\xi_{21}^{g_{i}}\right)^{*_{g}}\right) \quad$ using

(14) and (16), it follows that there exists $\eta_{j} \in C^{\infty}\left(\operatorname{Hom}\left(\left(T^{*} M\right)^{* j} \mathbb{R}\right)\right)$ such that $\sum_{i=1}^{\infty}\left(\Delta_{g_{i}}-\left(\Delta_{h_{i}}\right)^{* g_{i}}\right)=\sum_{i=1}^{\infty}\left(\eta_{0}+\eta_{1} \circ \nabla^{g_{i}}+\eta_{2} \circ\left(\nabla^{g_{i}}\right)^{2}\right)$ and these sections satisfy

$$
\sum_{i=1}^{\infty}\left|\eta_{j}\right|_{g_{i}}(x) \leq C \beta(x), 0 \leq j \leq 2, x \in M .
$$

By principle we have

$$
\begin{aligned}
& \sum_{i=1}^{\infty}\left(\mathrm{e}^{-t \Delta_{g_{i}}}-\mathrm{e}^{-t \Delta \Delta_{h_{i}}}\right)=\sum_{i=1}^{\infty}\left(\int_{0}^{t} \mathrm{e}^{-s \Delta_{g_{i}}}\left(\Delta_{h_{i}}-\Delta_{g_{i}}\right) \mathrm{e}^{-(t-s) \Delta_{h_{i}}} \mathrm{~d} s\right) \\
& =\sum_{i=1}^{\infty}\left(\int_{0}^{\frac{t}{2}} \mathrm{e}^{-s \Delta_{g_{i}}}\left(\Delta_{h_{i}}-\Delta_{g_{i}}\right) \mathrm{e}^{-(t-s) \Delta_{h_{i}}} \mathrm{~d} s+\int_{\frac{t}{2}}^{t} \mathrm{e}^{-s \Delta_{g_{i}}}\left(\Delta_{h_{i}}-\Delta_{g_{i}}\right) \mathrm{e}^{-(t-s) \Delta_{h_{i}}} \mathrm{~d} s\right)
\end{aligned}
$$

Using (22) and (23) we can proceed as above and prove that $\sum_{i=1}^{\infty}\left(\Delta_{g_{i}}-\left(\Delta_{h_{i}}\right)^{* g_{i}}\right) \mathrm{e}^{-t \Delta_{g_{i}}}$ is a trace class operator.

\section{Conflicts of Interest}

The authors declare no conflicts of interest regarding the publication of this paper.

\section{References}

[1] Itoa, K. and Skibsted, E. (2019) Time-Dependent Scattering Theory on Manifolds. Journal of Functional Analysis, 277, 1423-1468. https://doi.org/10.1016/j.jfa.2019.05.016

[2] Mishchenko, M.I. (2018) "Independent" and "Dependent" Scattering by Particles in a Multi-Particle Group. OSA Continuum, 1, 243-260. https://doi.org/10.1364/OSAC.1.000243

[3] Kaizuka, K. (2019) Scattering Theory for the Laplacian on Symmetric Spaces of Non-Compact Type and Its Application to a Conjecture of Strichartz. Journal of Functional Analysis, 276, 329-379. https://doi.org/10.1016/j.jfa.2018.11.005

[4] Cazenave, T. and Naumkin, I. (2018) Modified Scattering for the Critical Nonlinear Schrödinger Equation. Journal of Functional Analysis, 274, 402-432. https://doi.org/10.1016/j.jfa.2017.10.022

[5] de Aldecoa, R.T. (2020) Stationary Scattering Theory for Unitary Operators with an Application to Quantum Walks. Journal of Functional Analysis, 279, 108704 https://doi.org/10.1016/j.jfa.2020.108704

[6] Faupin, J. and Nicoleau, F. (2019) Scattering Matrices for Dissipative Quantum Systems. Journal of Functional Analysis, 277, 3062-3097. https://doi.org/10.1016/j.jfa.2019.06.010

[7] Behrndt, J., Malamud, M.M. and Neidhardt, H. (2017) Scattering Matrices and Dirichlet-to-Neumann Maps. Journal of Functional Analysis, 273, 1970-2025. https://doi.org/10.1016/j.jfa.2017.06.001

[8] Hempel, R., Post, O. and Weder, R. (2014) On Open Scattering Channels for Manifolds with Ends. Journal of Functional Analysis, 266, 5526-5583. 
https://doi.org/10.1016/j.jfa.2014.01.025

[9] Müller, W. and Salomonsen, G. (2007) Scattering Theory for the Laplacian on Manifolds with Bounded Curvature. Journal of Functional Analysis, 253, 158-206. https://doi.org/10.1016/j.jfa.2007.06.001

[10] Eichhorn, J. (1995) Spaces of Riemannian Metrics on Open Manifolds. Results in Mathematics, 27, 256-283. https://doi.org/10.1007/BF03322831

[11] Melrose, R.B. (1993) The Atiyah-Patodi-Singer Index Theorem. Vol. 4, A K Peters, New York. https://doi.org/10.1201/9781439864609

[12] Mazzeo, R. and Vasy, A. (2005) Analytic Continuation of the Resolvent of the Laplacian on Symmetric Spaces of Non-Compact Type. Journal of Functional Analysis, 228, 311-368. https://doi.org/10.1016/j.jfa.2004.10.003

[13] Strohmaier, A. (2005) Analytic Continuation of Resolvent Kernels on Non-Compact Symmetric Spaces. Mathematische Zeitschrift, 250, 411-425.

https://doi.org/10.1007/s00209-004-0760-y

[14] Cheeger, J., Gromov, M. and Taylor, M. (1982) Finite Propagation Speed, Kernel Estimates for Functions of the Laplace Operator, and the Geometry of Complete Riemannian Manifolds. Journal of Differential Geometry, 17, 15-53.

https://doi.org/10.4310/jdg/1214436699 